\title{
Ferramentas tecnológicas a serviço da avaliação no contexto de ensino-aprendizagem: um estudo comparativo
}

\section{Technological tools for teaching-learning assessment: a comparative study}

\author{
Gilmara Oliveira Maquiné \\ Instituto Federal de Educação, Ciência e Tecnologia do Amazonas \\ Iandra Maria Weirich da Silva Coelho \\ Instituto Federal de Educação, Ciência e Tecnologia do Amazonas
}

\begin{abstract}
Resumo: Este artigo tem como objetivo apresentar um conjunto de plataformas virtuais de ensino e ferramentas tecnológicas que permitem a avaliação do processo de ensinoaprendizagem no contexto virtual. Os procedimentos metodológicos envolvem uma pesquisa de cunho bibliográfico, identificação, levantamento e seleção de ferramentas tecnológicas, mediante uma análise comparativa, com foco qualitativo na descrição das principais contribuições e limitações. Os resultados apontam, entre as principais contribuições, a disponibilidade de diferentes recursos para a avaliação, a facilidade de uso de algumas ferramentas, a possibilidade de integração com outros ambientes, a exportação de artefatos avaliativos, o envio de feedback aos alunos, e compartilhamento de instrumentos de avaliação. Entre as principais limitações, o credenciamento junto às instituições, a dificuldade com o idioma e a necessidade de conhecimentos prévios para utilização de recursos.
\end{abstract}

Palavras-chave: Ferramentas tecnológicas. Plataformas virtuais de ensino. Avaliação.

\begin{abstract}
This article aims to present a set of virtual teaching platforms and technological tools that allow the evaluation of the teaching-learning process in the virtual context. The methodological procedures involve a bibliographic research, identification, survey and selection of technological tools, through a comparative analysis, with a qualitative focus on the description of the main contributions and limitations. The results point, among the main contributions, the availability of different resources for the evaluation, the ease of use of some tools, the possibility of integration with other environments, the export of evaluative artifacts, the sending of feedback to students, and the sharing of information assessment tools. Among the main limitations, accreditation with institutions, difficulty with the language and the need for prior knowledge to use resources.
\end{abstract}

Keywords: Technological tools. Virtual learning platforms. Assessment.

MAQUINÉ, Gilmara Oliveira; COELHO, Iandra Maria Weirich da Silva. Ferramentas tecnológiças a serviço da avaliação no contexto de ensino-aprendizagem: um estudo comparativo. Informática na Educação: teoria \& prática, Porto Alegre, v. 23, 


\section{Introdução}

Os constantes avanços tecnológicos e científicos ocorridos têm acarretado distintas mudanças que refletem, atualmente, tanto na sociedade como no âmbito educativo. As práticas têm sido alteradas em função das tecnologias digitais, "fazendo com que seja necessário repensar a prática pedagógica em sala de aula (e para além dela)" (MEDEIROS; MEDEIROS, 2018, p. 1).

Nesse cenário, de acordo com Machado (2012), a criação de plataformas reflete a maneira como as instituições buscam alcançar essas mudanças, principalmente na construção de conhecimentos, gerados em conjunto com o desenvolvimento tecnológico. "A vantagem principal deste tipo de aplicações está, sobretudo, no fato de aproveitar as competências digitais já adquiridas pelos jovens, procurando, ao mesmo tempo, aumentar a sua motivação para o uso destas no processo de ensino e aprendizagem" (MACHADO, 2012, p. 13).

O uso de plataformas educacionais tem crescido atualmente, além do fato de que "estamos na transformação para uma era digital, onde os alunos requerem o uso inovador de tecnologias para o aprendizado e/ou estão em situações em que são impossibilitados de ter aulas presenciais" (FUENTES PENNA; CRUZ SÁNCHEZ; DÍAZ DELGADO, 2019, p. 49).

Com base nesse cenário, evidenciamos a necessidade de novas propostas para inovar as práticas de ensino, aprendizagem e avaliação. Isso implica ressaltar a relevância do uso de diferentes ferramentas tecnológicas no contexto educacional, levando em conta os avanços registrados com o desenvolvimento das tecnologias digitais nos últimos anos, que evoluem de maneira exponencial nas mais diversas áreas da vida cotidiana (PASSERO; ENGSTER; DAZZI, 2016).

Nesse estudo, destacamos a importância dessas tecnologias digitais no processo avaliativo, considerando a necessidade de contar com "ferramentas e processos válidos e confiáveis de avaliação em distintos momentos da formação" (BARRA SALAZAR; MORA DONOSO, 2013, p.4). Tais ferramentas podem auxiliar o docente no acompanhamento do ensino-aprendizagem, com base nos resultados alcançados durante o processo, o qual envolve a aquisição e desenvolvimento de saberes, práticas, experiências, capacidades e comportamentos.

Tal constatação ressalta a necessidade de "conduzir simultaneamente mudanças profundas na avaliação educacional". Para Díaz e Hernández (2007, p. 350-351), "se as práticas de avaliação não forem modificadas, as supostas mudanças pedagógicas no ensino não terão impacto no sistema educacional ou nos alunos".

Para tanto ressaltamos a importância de novos processos avaliativos, principalmente em contextos virtuais de ensino, que podem incluir tanto a modalidade híbrida como a distância, em que são utilizadas distintas plataformas educativas para auxiliar na prática docente, especialmente, no acompanhamento e gerenciamento de dados e informações disponíveis sobre o processo formativo do estudante.

Diante do exposto, o artigo tem como objetivo apresentar um conjunto de ferramentas tecnológicas que permitem a avaliação no contexto de ensino virtual. Para alcançar esse objetivo, o percurso metodológico envolve uma pesquisa qualitativa exploratória sequencial, organizada 
em quatro etapas e adaptada a partir dos pressupostos de Cresswell (2010, p. 1): i) levantamento das ferramentas disponíveis na web; 2) seleção das ferramentas que compõem a amostra inicial; 3) delimitação do corpus de estudo; e 4) análise comparativa das ferramentas da amostra final.

Ainda com relação aos procedimentos metodológicos, neste estudo, utilizamos o método comparativo como um procedimento analítico central de análise, levando em conta que "a comparação é o instrumento apropriado em situações em que o número de casos em estudo é muito pequeno para permitir a utilização de análise estatística" (LIJPHART, 1971 apud PÉREZ LIÑÁN, 2010, p.1).

O presente artigo está organizado em quatro seções, iniciando com uma discussão sobre plataformas virtuais e o processo de avaliação, seguida pelo detalhamento dos procedimentos metodológicos, resultados e discussões e as considerações finais.

\section{Plataformas Virtuais e Avaliação}

Atualmente, as plataformas virtuais são amplamente utilizadas no âmbito educacional e o acesso ao mais diversos recursos dessas plataformas se tornou uma prática comum. "É cada vez mais comum o recurso a sistemas ou plataformas de gestão da aprendizagem, frequentemente designadas por plataformas de e-learning, ambientes virtuais de aprendizagem ou Web-Based Learning Environments (WBLE)" (MORAIS; CABRITA, 2007, p. 482).

Essas plataformas digitais podem auxiliar nas práticas pedagógicas "como estratégia capaz de desenvolver habilidades básicas e tecnológicas, que podem levar progressivamente à modificação do paradigma tradicional de ensino existente" (PÉREZ CERVANTES; BARROS SAKER, 2013, p. 69). Além disso, têm potencial para contribuir com o desenvolvimento de atividades dentro e fora da sala de aula.

Comumente, as plataformas de ensino costumam englobar uma "ampla gama de aplicativos de computador instalado em um servidor, cuja função é facilitar o corpo docente na criação, administração, gerenciamento e distribuição de Cursos na Internet" (SÁNCHEZ RODRÍGUEZ, 2009, p. 218). Inclusive, "quase todas incorporam elementos comuns, muito semelhantes, que fazem com que as semelhanças entre eles sejam mais numerosas do que as diferenças" ( ibdem).

De acordo com Sánchez Rodríguez (2009), essas plataformas podem ser utilizadas para atividades de e-learning, apoiar as atividades presenciais e ser utilizadas no ensino semipresencial. Além disso, entre os recursos disponibilizados, é possível citar as ferramentas de distribuição de conteúdo, as ferramentas de comunicação e colaboração assíncrona e síncrona, as ferramentas de administração e as ferramentas de avaliação.

Para Gabardo, Quevedo e Ulbricht (2010, p.67), as diversas plataformas disponíveis facilitam a criação de ambientes de aprendizagem. "Nelas, estão embutidos contornos tecnológicos e pedagógicos para o desenvolvimento de metodologias educacionais, utilizando canais de interação Web aptos a oferecer suporte para atividades educacionais de forma virtual". 
O uso dessas plataformas digitais proporciona aos usuários diversas possibilidades, entre elas, ampliar a autonomia, "na medida em que amplia o espaço decisório dos estudantes, em que a busca, a compreensão e a avaliação de fontes necessárias podem ocorrer sem a presença do docente" (COSTA; FRANCO, 2007, p.5). Além disso, o uso dessas plataformas pode possibilitar "que alunos e professores desenvolvam um processo de ensino-aprendizagem capaz de abranger qualquer dimensão de aprendizagem do aluno, seja no modelo a distância ou presencial" (BRITO et al.,2015, p. 4).

Outro aspecto refere-se à agilidade na realização de procedimentos on-line e de "avaliações através de questionários ou ferramentas simuladas hospedadas em servidores na web" (GONZÁLEZ-RUIZ, 2018, p.28).

Nesse cenário, destacamos diferentes plataformas de ensino virtual e ferramentas tecnológicas que podem auxiliar no processo avaliativo nas modalidades híbrida, presencial ou a distância, levando em conta suas principais contribuições e limitações.

Vale ressaltar que essas plataformas de ensino

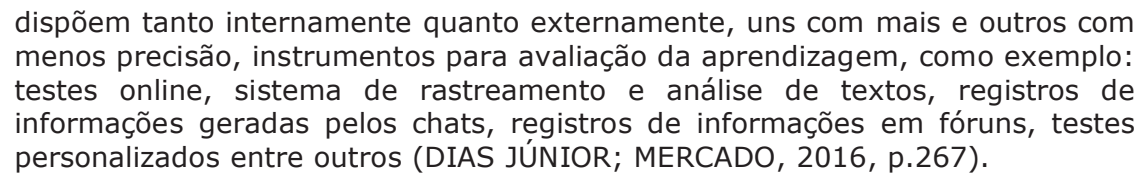

Apesar da disponibilidade de distintos instrumentos avaliativos, vale ressaltar que "mesmo que o aluno tenha realizado todas as atividades propostas para o ambiente virtual, ele é aprovado ou reprovado a partir do resultado obtido na prova final presencial" (SILVA; LEITE; SILVA, 2009, p. 242), tendo em vista a obrigatoriedade de momentos presenciais para a realização da avaliação dos estudantes, conforme previsto pela legislação brasileira. Segundo as autoras, esse tipo de abordagem avaliativa tradicional, ancorada em provas presenciais, atribui um peso maior a essas provas, destacando "a necessidade de desenvolvimento e implementação de metodologias de avaliação inovadoras e que sejam apropriadas [...]" (SILVA; LEITE; SILVA, 2009, p. 242).

Tais constatações evidenciam a necessidade de "contemplar aspectos relacionados a técnicas/instrumentos de avaliação, critérios de julgamento, estratégias de aprendizagem utilizadas pelos alunos, devolução de resultados e autoavaliação de alunos e de professores/tutores" (SILVA; LEITE; SILVA, 2009, p. 242).

Nesse enquadramento, por meio da avaliação podemos coletar "indicativos da aprendizagem e formular avaliações acerca do progresso dos alunos", bem como valorar os "resultados esperados da aprendizagem" (BERROCOSO; DOMÍNGUEZ; SÁNCHEZ, 2012, p. 51). Avaliar esse processo leva em conta a valoração dos desempenhos alcançados pelos estudantes que podem ser materializados em formato de produtos, ações ou tarefas desenvolvidas, por meio de execuções concretas e observáveis, que podem ser avaliadas com base nos saberes, habilidades e comportamentos. 
Vale destacar que esses elementos combinados são utilizados pelos indivíduos para enfrentar diversas situações, como as profissionais, escolares, da vida cotidiana, entre outras (PERRENOUD et al., 2002), integrando diferentes tipos de competências. Assim, apesar de o conceito de competências ser considerado "indiscutivelmente complexo", cuja "diversidade decorre da panóplia de perspectivas teóricas existentes" (ANDRÉ; RODRIGUES, 2019, p. 43), compreendemos esse conceito, neste estudo, em "uma dimensão ampla contemplando não só aspectos racionais, cognitivos ou mentais, mas também processos intersubjetivos, afetivos, socioculturais [...]" (MARINHO-ARAUJO; RABELO, 2015, p. 448).

Assim, diferentes tipos de saberes, habilidades e atitudes dos estudantes integram diferentes tipos de competências, entre elas a digital, necessária para a utilização efetiva das plataformas e distintas ferramentas tecnológicas.

\begin{abstract}
As competências digitais na sociedade do século 21 são cada vez mais necessárias para o desenvolvimento de atividades relacionadas com educação e pesquisa; o acesso à informação é hoje, em dia, um requisito indispensável cujas fontes de informação se orientam ao acesso digital [...] portanto, exigem o uso constante dessas competências (FUENTES PENNA; CRUZ SÁNCHEZ; DÍAZ DELGADO, 2019, p. 47, tradução nossa).
\end{abstract}

O desenvolvimento de tais competências pode ser potencializado com o uso de diferentes ferramentas tecnológicas que também podem contribuir para valorar as aprendizagens alcançadas e auxiliar no processo avaliativo. Algumas dessas vantagens já foram destacadas na literatura, tais como: distribuição de provas sem a necessidade de papel, padronização no gerenciamento de provas, análise e interpretação dos resultados da avaliação, possibilidade de utilizar ferramentas com perguntas interativas, motivação dos estudantes, na perspectiva da concentração e rendimento, entre outras (GRACIA; ORTIZ, 2014).

\footnotetext{
Uma das grandes vantagens que o processo de ensino-aprendizagem em AVA tem no campo da avaliação é, sem dúvida, a possibilidade de feedback quase imediato ao estudante. Além disso, pode-se destacar também a flexibilidade de datas, o respeito ao ritmo individual, a abordagem modular e o redimensionamento das noções de tempo e espaço (SILVA; LEITE; SILVA, 2009, p. 243).
}

Com base nessas considerações destacamos, a seguir, os procedimentos metodológicos para a realização do estudo comparativo de diferentes plataformas e ferramentas tecnológicas que podem auxiliar professores e estudantes na avaliação do processo de ensino-aprendizagem, especialmente, em contextos virtuais.

Cabe destacar que outros estudos já foram realizados tendo como temática as plataformas de ensino-aprendizagem, por meio do método comparativo (GABARDO; QUEVEDO; ULBRICHT, 2010; FUENTES PENNA; CRUZ SÁNCHEZ; DÍAZ DELGADO, 2019) e que enfatizam características como a aprendizagem colaborativa, interatividade, multimídia, usabilidade e acessibilidade das principais plataformas utilizadas. 


\section{Procedimentos Metodológicos}

O presente estudo foi realizado a partir de uma pesquisa qualitativa exploratória, organizada em quatro etapas, a partir dos pressupostos de Cresswell (2010), sendo elas: 1) levantamento das ferramentas disponíveis na web; 2 ) seleção das ferramentas que compõem a amostra inicial; 3) delimitação da amostra de análise; e 4) análise comparativa das ferramentas da amostra final.

Como metodologia, utilizamos o método comparativo como um procedimento analítico central de análise (PÉREZ LIÑÁN, 2010). Nesse caso, a investigação procede a partir da seleção de uma amostra, com um número limitado de casos que permitiu a viabilidade das análises. Segundo Pérez Liñán (2010), uma estratégia clássica para definir tal amostra em um estudo comparativo consiste em selecionar casos paradigmáticos do fenômeno que se deseja investigar, por meio da estratégia de semelhança e diferença.

No presente estudo, exploramos as características comuns, por meio do método da semelhança, levando em conta indicadores avaliados em um conjunto de estudos, oriundos de bases científicas. A amostra inicial compreende a identificação e análise de vinte e nove (29) ferramentas, organizadas por meio de um levantamento bibliográfico, realizado em bases de dados e repositórios, tais como: Google Scholar, Dialnet, Scielo, Latindex, Redalyc, entre outras, tendo como expressões de busca as seguintes palavras-chave: Ferramentas para avaliação, Herramientas para la evaluación, evaluation tool, assessment tool.

Vale destacar que foram incluídos na análise final, apenas os softwares livres, considerando a perspectiva proposta pela Free Software Foundation em que "os usuários possuem a liberdade de executar, copiar, distribuir, estudar, mudar e melhorar o software" (GNU, 2019).

Para definir essa amostra utilizamos a técnica de amostragem não probabilística, empregada pela falta de conhecimento do quantitativo total de ferramentas tecnológicas disponíveis. Cabe ressaltar que a razão para essa amostragem não probabilística deve-se à falta de necessidade de que os dados evidenciem precisamente o quantitativo total de ferramentas existentes, já que não é o propósito da pesquisa. Além disso, não há intenção de generalizar os dados obtidos, não sendo, portanto, necessário o uso de uma amostragem mais ou menos representativa da população investigada, sendo impossível examinar todos os elementos da população.

A amostra inicial compreende ferramentas digitais, ambientes virtuais de aprendizagem, metodologias, modelos, instrumentos e plataformas, que podem ser utilizadas para realizar 0 processo avaliativo. As ferramentas identificadas durante a fase da amostra inicial estão presentes no Quadro 1. 


\begin{tabular}{|c|c|}
\hline Ferramenta & Descrição \\
\hline A2COMP & $\begin{array}{l}\text { Produto de Mestrado, possibilita a criação de um plano de ação para o docente, além de } \\
\text { proporcionar o diagnóstico inicial dos alunos (PRIMO, 2004). }\end{array}$ \\
\hline Ágora Virtual & $\begin{array}{l}\text { Plataforma voltada para o ensino-aprendizagem, lançada em 2006. Reúne em seu } \\
\text { escopo Fóruns, Questionários e outros recursos para a aprendizagem. Link para acesso: } \\
\text { https://agoravirtual.net/index_en.php }\end{array}$ \\
\hline Amadeus & $\begin{array}{l}\text { Ambiente virtual de aprendizagem criado pelo Grupo de Ciências Cognitivas e Tecnologia } \\
\text { Educacional, da Universidade Federal de Pernambuco, e lançado em 2007. Possibilita a } \\
\text { criação de Fóruns, Questionário, Tarefas e Trabalhos com Revisão. Link para acesso: } \\
\text { https://softwarepublico.gov.br/social/amadeus }\end{array}$ \\
\hline AVACOMP & $\begin{array}{l}\text { Produto da dissertação de Mestrado de Brandão (2012). Foi criado para uma instituição } \\
\text { particular e é de uso restrito, realiza a avaliação por meio de rubricas, mapeamento de } \\
\text { níveis de proficiência e desempenho dos alunos. }\end{array}$ \\
\hline DHIN & $\begin{array}{l}\text { A metodologia foi desenvolvida por Núnez Rojas (2007) e tem como foco o } \\
\text { desenvolvimento de competências investigativas nos alunos, propondo um modelo para } \\
\text { avaliação de competências. }\end{array}$ \\
\hline Digimina & $\begin{array}{l}\text { Ferramenta web para autoavaliação de competências digitais docentes de Escolas da } \\
\text { Estônia. Link para acesso: http://digimina.opetaja.ee/ }\end{array}$ \\
\hline DINNO & $\begin{array}{l}\text { Software para criação de um plano de ação para inovação no processo avaliativo, criado } \\
\text { em } 2014 \text {, por Ibarra Sáiz e Rodríguez Gómez. }\end{array}$ \\
\hline Dipeval & $\begin{array}{l}\text { Ferramenta para criação de procedimentos de avaliação, criada em 2010, por Ibarra } \\
\text { Sáiz e Rodríguez Gómez. Traz em seu escopo os seguintes recursos para apoiar a } \\
\text { avaliação: Escala de valoração, Instrumento misto, Lista de controle, Rubrica. }\end{array}$ \\
\hline $\begin{array}{l}\text { Edu Web/Aula } \\
\text { Net }\end{array}$ & $\begin{array}{l}\text { Ambiente virtual de aprendizagem, desenvolvido pelo Laboratório de Engenharia de } \\
\text { Software - LES - do Departamento de Informática da PUC-Rio e lançado em 1998. Faz } \\
\text { uso de Fóruns, Questionários, Tarefas e Revisão de pares para auxiliar no processo de } \\
\text { avaliação. Link para acesso: http://www.eduweb.com.br/elearning_tecnologia.asp }\end{array}$ \\
\hline ELO & $\begin{array}{l}\text { Sistema de autoria criado por Leffa (2013), que disponibiliza e compartilha materiais } \\
\text { para o ensino de línguas, auxiliando na criação de atividades, sequências didáticas e } \\
\text { quizzes. }\end{array}$ \\
\hline E-ProInfo & $\begin{array}{l}\text { Ambiente virtual de aprendizagem, criado pelo Laboratório de Tecnologia da Informação } \\
\text { e Mídias Educacionais (LabTime), da Universidade Federal de Goiás (UFG) em parceria } \\
\text { com o Ministério da Educação (MEC) atendendo ao Programa Nacional de Tecnologia } \\
\text { Educacional (ProInfo). Foi lançado em 1997, e possui as seguintes funcionalidades que } \\
\text { auxiliam no processo avaliativo: Controle de notas. Link para acesso: http://e- } \\
\text { proinfo.mec.gov.br/ }\end{array}$ \\
\hline Eureka & $\begin{array}{l}\text { Ambiente virtual de aprendizagem criado pela Pontifícia Universidade Católica (PUC) do } \\
\text { Estado do Paraná e lançado em 1998. Possibilita a criação do Plano de trabalho, } \\
\text { configuração de agendas e criação de Fóruns. Link para acesso: https://eureka.pucpr.br/ }\end{array}$ \\
\hline EvalComix & $\begin{array}{l}\text { Serviço da Web para avaliação eletrônica integrável em sistemas de gerenciamento de } \\
\text { aprendizagem. Criado em 2007, pelo Grupo de Pesquisa EVALfor, da Universidade de } \\
\text { Cádiz. Link para acesso: http://evalcomix.uca.es/ }\end{array}$ \\
\hline $\begin{array}{lr}\text { Ferramenta } & \text { para } \\
\text { avaliação } & \text { de } \\
\text { competência }\end{array}$ & $\begin{array}{l}\text { Publicado em 2004, por Roque, Elia e Motta. Possibilita a informação de competências, } \\
\text { associação de habilidades relacionadas às competências e critérios de avaliação, } \\
\text { relacionando-as a situações-problema (ROQUE; ELIA; MOTTA, 2004). }\end{array}$ \\
\hline $\begin{array}{lr}\text { Ferramenta } & \text { para } \\
\text { estimar } & \text { a } \\
\text { proficiência } & \end{array}$ & $\begin{array}{l}\text { Oriunda da dissertação de Mestrado de Coutinho (2013). Possui um teste baseado na } \\
\text { Teoria de Resposta ao Item, propondo uma análise estatística dos resultados. }\end{array}$ \\
\hline
\end{tabular}




\begin{tabular}{|c|c|}
\hline Goobric & $\begin{array}{l}\text { Plugin criado em 2013, pela empresa Cloudlab que possibilita a criação de rubricas para } \\
\text { serem utilizadas em avaliação. }\end{array}$ \\
\hline $\begin{array}{l}\text { Google } \\
\text { Classroom }\end{array}$ & $\begin{array}{l}\text { Ambiente virtual de aprendizagem criado e mantido pela empresa Google e lançado em } \\
\text { 2014. Possui recursos que auxiliam na prática docente, tais como: tarefas, Postagens, } \\
\text { Compartilhamento de vídeos, links, Perguntas e Avisos. A partir da atualização } \\
\text { disponibilizada no final de } 2019 \text {, o ambiente passou a provê a criação/associação de } \\
\text { rubricas nas atividades de aprendizagem. }\end{array}$ \\
\hline InevalCo & $\begin{array}{l}\text { Ambiente criado em 2007, pelo Grupo de Pesquisa EVALfor, da Universidade de Cádiz, } \\
\text { no qual disponibiliza os seguintes recursos que apoiam a avaliação: Listas de controle, } \\
\text { Escalas de estimativa e Rubricas. Link para acesso: http://inevalco.uca.es/ }\end{array}$ \\
\hline $\begin{array}{ll}\text { Instrumento } & \text { de } \\
\text { Avaliação } & \text { de } \\
\text { Rubrica } & \end{array}$ & $\begin{array}{l}\text { Resultado do trabalho de Velozo (2014). Emprega a rubrica como instrumento de } \\
\text { avaliação de competências. }\end{array}$ \\
\hline Learning Space & $\begin{array}{l}\text { Criado pela empresa The Open University em 2006, possui funcionalidades que apoiam } \\
\text { a autoavaliação e criação de fóruns. Link para acesso: } \\
\text { https://www.elearninglearning.com/learning-space/lms/ }\end{array}$ \\
\hline $\begin{array}{ll}\text { Modelo } & \text { de } \\
\text { Avaliação } & \text { de } \\
\text { Competências } & \end{array}$ & $\begin{array}{l}\text { O Modelo de Avaliação de Competências (Assessment of competencies model) foi } \\
\text { desenvolvido no trabalho de Cardona, Velez e Tobón (2013). Propõe um modelo que } \\
\text { auxilia no estabelecimento de competências, desenho de critérios para avaliação, } \\
\text { estabelecimento de evidências, criação de rubricas e avaliação. }\end{array}$ \\
\hline $\begin{array}{l}\text { Modelo de } \\
\text { Avaliação por } \\
\text { competências } \\
\text { para EAD }\end{array}$ & $\begin{array}{l}\text { Foi proposto por Roque (2004) e oportuniza o mapeamento das competências e } \\
\text { habilidades a serem desenvolvidas, definição dos critérios orientadores para o } \\
\text { desenvolvimento das competências visadas, escolha de uma estratégia avaliativa, e } \\
\text { avaliação para verificação do alcance das competências. }\end{array}$ \\
\hline Moodle & $\begin{array}{l}\text { É um software livre para apoiar o ensino, criado em 2001, e vem sendo utilizado por } \\
\text { diversas instituições de ensino-aprendizagem para propagar a educação a distância. }\end{array}$ \\
\hline MOSAICO & $\begin{array}{l}\text { Modelo proposto no trabalho de Roque, Elia e Motta (2006) que descreve o processo } \\
\text { para mapeamento de Competências, Habilidade e aspectos a serem avaliados, utilização } \\
\text { de Rubrica. }\end{array}$ \\
\hline Rooda & $\begin{array}{l}\text { Criado em 2004, pela Universidade Federal do Rio Grande do Sul. As funcionalidades } \\
\text { que compõem o sistema são: anotações, sistema automático de notas. Link para acesso: } \\
\text { https://ead.ufrgs.br/rooda/ }\end{array}$ \\
\hline SACHA & Sistema de Avaliação por Conhecimentos, Habilidades e Atitudes (FEITOSA, 2009). \\
\hline SWAD & $\begin{array}{l}\text { Ambiente Virtual de Aprendizagem, criado pela Universidade de Granada. Inclui em seu } \\
\text { escopo os seguintes recursos: Atividades, Projetos, Testes de autoavaliação, Pesquisas, } \\
\text { Chamadas de exames e Arquivos do estudante. Link para acesso: https://swad.ugr.es }\end{array}$ \\
\hline TelEduc & $\begin{array}{l}\text { Desenvolvido pelo Núcleo de Informática Aplicada à Educação, do Instituto de } \\
\text { Computação da Unicamp, no ano de 1998. É possível criar atividade, avaliações, diário } \\
\text { de bordo, além de fóruns de discussão e Portfólios. Link para acesso: } \\
\text { http://teleduc4.multimeios.ufc.br/ }\end{array}$ \\
\hline $\begin{array}{l}\text { Web } \\
\text { Creator }\end{array}$ & Criado por Vieira, em meados de 2008. Possibilita a realização de avaliações on-line. \\
\hline
\end{tabular}

Fonte: Elaborado pelas autoras (2020).

A partir dessa amostra inicial, foram identificados casos paradigmáticos do fenômeno de interesse. Para tanto, utilizamos o denominado método de desenho baseado nos casos mais diferentes, que segundo Pérez Liñán (2010), vão além da semelhança das características, com 
respeito às variáveis de controle. Nesse caso, elencamos como indicadores diferenciais: i) se são ferramentas digitais; ii) se estão disponíveis para acesso gratuito via web; iii) se permitem avaliação de competências e habilidades.

Vale ressaltar que esse último aspecto leva em conta a necessidade de tratar sobre a avaliação das competências, a partir das orientações da Base Nacional Comum Curricular (BNCC), aprovada em 2017, que estabelece conhecimentos, competências e habilidades que se espera que todos os estudantes desenvolvam ao longo da escolaridade básica. A inclusão desse diferencial também justifica-se na tentativa de promover novas práticas, com foco no desenvolvimento de competências, com o objetivo de ressignificar estruturas, programas acadêmicos, práticas de ensino, aprendizagem e avaliação (DECLARAÇÃO DE BOLONHA, 1999).

A partir dessa estratégia metodológica, reunimos no Quadro 2 as ferramentas que foram retiradas da amostra de análise.

Quadro 2 - Descrição dos indicadores diferenciais das ferramentas.

\begin{tabular}{|c|c|}
\hline \multicolumn{2}{|l|}{ é uma ferramenta digital? } \\
\hline $\begin{array}{l}\text { Considera se a ferramenta tem uma interface } \\
\text { digital para realização das atividades. "Ambientes } \\
\text { para que o aluno acesse a qualquer momento e } \\
\text { possa desenvolver seu conhecimento" (KLESMAN; } \\
\text { REATEGUI; RAPKIEWICZ, 2011, p.1101). }\end{array}$ & $\begin{array}{l}\text { A2COMP; Avaliação mediada por Rubrica (VELOZO) } \\
\text { DHIN; Modelo de Avaliação de Competências } \\
\text { (Assessment of competencies model); Modelo de } \\
\text { Avaliação por competências para EAD; MOSAICO. }\end{array}$ \\
\hline \multicolumn{2}{|l|}{ está disponível na web? } \\
\hline $\begin{array}{l}\text { Identificadas em dissertações de Mestrado e } \\
\text { artigos, porém estavam indisponíveis durante o } \\
\text { período da análise. }\end{array}$ & $\begin{array}{l}\text { AVACOMP (BRANDÃO, 2012), Ferramenta para } \\
\text { avaliação de competência (ROQUE; ELIA; MOTA, } \\
\text { 2004), Ferramenta para estimar a proficiência } \\
\text { (COUTINHO, 2013) e DigiMina. }\end{array}$ \\
\hline \multicolumn{2}{|c|}{ permite a avaliação de competências e habilidades? } \\
\hline $\begin{array}{l}\text { Considera aspectos que compõem a avaliação de } \\
\text { competências: acompanhamento r do } \\
\text { desenvolvimento na competência definida, } \\
\text { feedback, autoavaliação. }\end{array}$ & $\begin{array}{l}\text { Amadeus; Edu Web/Aula Net; ELO; E-ProInfo; Eureka; } \\
\text { Learning Space; Rooda; TelEduc; WebQuest Creator. }\end{array}$ \\
\hline
\end{tabular}

Fonte: Elaborado pelas autoras (2020).

Após esses procedimentos, foram selecionadas nove ferramentas para a realização do estudo comparativo. Vale ressaltar que consideramos essa amostra adequada, tendo em vista os critérios propostos por Gil (2008), que identifica como uma amostra ideal para análise entre 4 e 10 objetos de pesquisa. Além disso, no âmbito de estudos comparativos, o número de casos analisados usualmente varia entre dois e vinte (PÉREZ LIÑÁN, 2010) e as estratégias para selecionar os casos relevantes têm variado ao longo do tempo (GERRING, 2007).

Inicialmente, evidenciamos, no Quadro 3, as nove ferramentas analisadas e as principais informações referentes aos autores, endereço de acesso e principais recursos que podem auxiliar no processo avaliativo de conhecimentos, habilidades e atitudes dos alunos, com relação às atividades a serem desenvolvidas no ensino-aprendizagem virtual. 
Quadro 3 - Ferramentas tecnológicas que apoiam o processo de avaliação.

\begin{tabular}{|c|c|c|c|}
\hline Ferramenta & $\begin{array}{l}\text { Autor/Universidad } \\
\text { e/Ano de criação }\end{array}$ & Endereço eletrônico & $\begin{array}{l}\text { Recursos que apoiam o processo } \\
\text { de avaliação de competências }\end{array}$ \\
\hline Ágora Virtual & $\begin{array}{l}\text { José Alfonso Accino/ } \\
\text { Universidad de } \\
\text { Málaga/ } 2006\end{array}$ & $\begin{array}{l}\text { http://www.agoravirtual. } \\
\text { es }\end{array}$ & Fóruns, Tarefas, Rubricas. \\
\hline DINNO & $\begin{array}{l}\text { Marisol Ibarra Sáiz; } \\
\text { Gregorio Rodríguez } \\
\text { Gómez/ Grupo de } \\
\text { Investigación EVALfor } \\
\text { - Universidade de } \\
\text { Cádiz/2014 }\end{array}$ & http://dinno.evalfor.net/ & $\begin{array}{l}\text { Plano de ação para inovação no } \\
\text { processo avaliativo. }\end{array}$ \\
\hline Dipeval & $\begin{array}{l}\text { Marisol Ibarra Sáiz; } \\
\text { Gregorio Rodríguez } \\
\text { Gómez/ Grupo de } \\
\text { Investigação EVALfor } \\
\text { - Universidade de } \\
\text { Cádiz/2010 }\end{array}$ & http://dipeval.uca.es/ & $\begin{array}{l}\text { Escala de valoração, Instrumento } \\
\text { misto, Lista de controle, Rubrica. }\end{array}$ \\
\hline EvalComix & $\begin{array}{l}\text { Grupo de } \\
\text { Investigação EVALfor } \\
\text { - Universidade de } \\
\text { Cádiz/2007 }\end{array}$ & http://evalcomix.uca.es/ & $\begin{array}{l}\text { Escala de valoração, Lista de } \\
\text { controle e escala de valoração, Lista } \\
\text { de controle, Rubrica, Instrumento } \\
\text { misto, Avaliação argumentativa. }\end{array}$ \\
\hline Goobric & CloudLab/2013 & $\begin{array}{l}\text { http://cloudlab.newvisio } \\
\text { ns.org/add-ons/goobric }\end{array}$ & Rubrica. \\
\hline $\begin{array}{l}\text { Google } \\
\text { Classroom }\end{array}$ & Google & $\begin{array}{l}\text { https://classroom.google } \\
\text {.com/ }\end{array}$ & Rubrica. \\
\hline InevalCo & $\begin{array}{l}\text { Grupo de } \\
\text { Investigação EVALfor } \\
\text { - Universidade de } \\
\text { Cádiz/2007 }\end{array}$ & http://inevalco.uca.es/ & $\begin{array}{l}\text { Listas de controle, Escalas de } \\
\text { estimativa, Rubricas. }\end{array}$ \\
\hline Moodle & $\begin{array}{l}\text { Martin } \\
\text { Dougiamas/2001 }\end{array}$ & https://moodle.org & $\begin{array}{l}\text { Formulários, Fóruns, } \\
\text { Atividade online, Tarefas, Wiki, } \\
\text { Rubricas. }\end{array}$ \\
\hline SWAD & $\begin{array}{l}\text { Universidade de } \\
\text { Granada/1999 }\end{array}$ & https://swad.ugr.es/pt & $\begin{array}{l}\text { Atividades, Projetos, Testes de } \\
\text { autoavaliação, Pesquisas, Chamadas } \\
\text { de exames, Arquivos do estudante. }\end{array}$ \\
\hline
\end{tabular}

Fonte: Elaborado pelas autoras (2020).

Para a análise dessas ferramentas selecionadas, consideramos dois critérios: técnicos e teóricos. Segundo Sabota e Pereira (2017, p.51), esses aspectos abrangem "a qualidade do material, a garantia do acesso a um grande número de aprendizes e de professores, bem como o impacto que a ferramenta traria na vida do usuário". Os aspectos técnicos referem-se às características funcionais da ferramenta, que incluem questões de usabilidade, acesso gratuito e suporte. Com relação aos teóricos, foram avaliados os conceitos que envolvem o processo de 
ensino-aprendizagem, tais como a mediação e avaliação das atividades, com foco nas competências e habilidades.

\section{Resultados e discussão}

Neste trabalho, consideramos os seguintes aspectos técnicos: facilidade de uso de algumas ferramentas, acesso gratuito, integração com diferentes ambientes e possibilidade de exportar artefatos avaliativos. Entre os aspectos teóricos, consideramos instrumentos e recursos que permitem a avaliação de conhecimentos, habilidades e atitudes dos estudantes.

Por meio da análise das ferramentas tecnológicas, buscamos identificar as principais características, potencialidades e possíveis limitações no processo avaliativo. Entre as principais contribuições destacamos: recursos disponíveis para a avaliação de diferentes tipos de saberes, habilidades e atitudes dos estudantes, facilidade de uso, possibilidade de integração com outros ambientes, exportação de artefatos avaliativos, envio de feedback aos alunos e compartilhamento de instrumentos de avaliação.

Com relação às potencialidades das ferramentas, destacamos primeiramente a facilidade de uso, considerando que todas são voltadas para o ensino-aprendizagem e apresentam termos comuns. Essa característica se relaciona com a heurística de usabilidade "correspondência entre o sistema e o mundo real", pois faz uso de frases, palavras e conceitos familiares ao usuário (NIELSEN; LORANGER, 2007). Apesar da facilidade apontada, vale destacar que as ferramentas Dipeval e EvalComix não apresentam opções de navegação ou botões para realizar ações como: salvar ou exportar. Esse aspecto pode ser uma barreira à sua utilização, pois a usabilidade considera "a interação dos usuários com os sistemas e não somente aspectos funcionais que impactam somente na interface" (MARQUES; BARBOSA; CONTE, 2016, p.1). Das oito ferramentas analisadas, podem ser consideradas de fácil utilização: Moodle, EvalComix, Dipeval, InevalCo.

Vale ressaltar que, de maneira, geral, os recursos mais frequentes que apoiam a avaliação de diferentes competências e habilidades são: rubricas, escala de valoração, lista de controle, fóruns, testes de autoavaliação. Tais recursos podem auxiliar na efetivação de novas propostas, com base em uma avaliação qualitativa, com foco na descrição da qualidade das aprendizagens e do progresso e desempenhos alcançados pelos estudantes, em diferentes atividades e projetos desenvolvidos no âmbito escolar.

Entre as ferramentas que disponibilizam tais recursos, destacamos a Dipeval que possibilita, no momento da criação da tarefa de avaliação, o detalhamento de instrumentos adequados a cada prática avaliativa, podendo contribuir com a aprendizagem dos estudantes envolvidos.

Em EvalComix, identificamos a possibilidade de criar diferentes dimensões dentro de uma mesma prática avaliativa, como por exemplo no uso de rubricas, a possibilidade de incluir diferentes critérios e descritores para qualificar a avaliação.

Com relação à integração com outros ambientes, identificamos a possibilidade no EvalComix de integração com o Moodle, possibilitando, por exemplo o uso de videoconferências. Exportar artefatos avaliativos em diferentes formatos, incluindo .PDF, são recursos disponíveis em 
EvalComix, Dipeval e InevalCo. A criação e compartilhamento de instrumentos de avaliação são destaques nas ferramentas Dipeval, EvalComix, e InevalCo. Quanto ao envio de feedback aos alunos, identificamos que as ferramentas Goobric, Moodle e SWAD apresentam esse recurso como apoio à aprendizagem do aluno.

Outro ponto positivo são os relatórios disponibilizados por algumas dessas ferramentas. Ágora Virtual, EvalComix, Goobric, Moodle e SWAD oferecem relatórios que possibilitam o gerenciamento do desempenho dos estudantes, a partir de dados quantitativos, tais como quantidade de tarefas realizadas, notas obtidas, quantidade de acessos, entre outros. No entanto, vale ressaltar a relevância de potencializar recursos que permitam a criação e acompanhamento de relatórios qualitativos em que seja possível ampliar os descritores referentes às aprendizagens desenvolvidas.

Especificamente para a avaliação de competências, destacamos o Moodle. Essa possibilidade foi integrada ao software em meados de 2014, a partir da Versão 3.1, e possibilita a criação de frameworks de competências, associados aos planos de aprendizagem que devem ser previamente criados e configurados no ambiente. Porém, para que o professor realize essa ação, é necessário que o mesmo tenha permissões de administrador do sistema e também receba capacitação direcionada ao uso dessa funcionalidade.

Identificamos ainda que no segundo semestre de 2019, o Google Classroom recebeu uma atualização que proporciou aos professores, a criação e reutilização de rubricas em suas respectivas tarefas. Tal recurso ficou disponível inicialmente na versão de testes para administradores e professores que preencheram um formulário de interesse para acesso a essa versão preliminar (GOOGLE, 2019). Faz-se necessário aprofundar os estudos para identificar as características detalhadas do recurso.

Por outro lado, entre as principais limitações identificadas nas ferramentas analisadas, destacamos: credenciamento junto às instituições, idioma, necessidade de impressão de materiais, investimento em equipamentos de hardware, uso exclusivo em determinados navegadores e conhecimentos prévios para utilização. A necessidade de credenciamento junto às instituições que gerenciam as ferramentas, como no caso de Ágora Virtual e SWAD, pode ser considerada uma limitação, na perspectiva do docente que necessita utilizar a ferramenta de maneira ágil e prática. As limitações quanto ao idioma referem-se ao fato de que a maioria das ferramentas, assim como seus manuais e tutoriais, estão em língua inglesa e espanhola, e isso pode ser um fator que limita a utilização da ferramenta, tendo em vista uma eventual falta de proficiência em outras línguas (tanto por parte do professor quanto dos alunos).

A impressão faz referência à necessidade de imprimir as escalas de valoração para serem utilizadas durante o processo avaliativo, em ferramentas como Dipeval. Outro fator que pode limitar o uso das ferramentas, considerando aspectos econômicos, refere-se ao investimento em infraestrutura de hardware para hospedagem do sistema, identificado na ferramenta EvalComix e Moodle. Além disso, no caso do Moodle, outros aspectos podem ser evidenciados como a necessidade de contratação de pessoal especializado para gerenciamento dessa plataforma. 
Quanto ao uso em navegadores exclusivos, identificamos que a ferramenta Goobric funciona apenas no Google Chrome, e para ter o uso de maneira completa, é necessário fazer uso de outras ferramentas como Octopus e Google Sheets. Quanto à necessidade de conhecimentos prévios para as ferramentas Goobric, Moodle, Ágora Virtual, faz-se necessário o usuário realizar treinamento para manusear os recursos de maneira adequada. Santos (2012) também aponta como uma das desvantagens na utilização do Moodle, a necessidade de desenvolver conhecimento para utilização plena das funcionalidades.

Com base nessas limitações, corroboramos os resultados do estudo comparativo realizado por autores como Gabardo, Quevedo e Ulbricht (2010), com relação à configuração apresentada pelas diferentes ferramentas tecnológicas, que apesar dos diferenciais e de novas propostas que podem auxiliar na avaliação, as desvantagens demonstram a importância de melhorias e otimização do potencial dessas ferramentas, tanto do ponto de vista tecnológico, quanto pedagógico.

\section{Considerações Finais}

Entre as principais contribuições desse estudo, destacamos a apresentação de um conjunto de ferramentas tecnológicas que podem potencializar o processo avaliativo, podendo auxiliar professores na seleção e uso de tecnologias que podem contribuir para o planejamento e avaliação de conhecimentos, habilidades ou atitudes de estudantes.

Entre as vantagens das ferramentas analisadas, destacamos a disponibilidade de recursos que podem ser usados por professores no processo avaliativo, tais como: rubrica, teste de autoavaliação, escala de valoração, lista de controle, fóruns, gerenciamento, análise e socialização dos dados referentes ao desempenho e aprendizagens desenvolvidas pelos estudantes, possibilidade de integração de algumas ferramentas com outros ambientes, realização de exportação de artefatos avaliativos, envio de feedback aos alunos, compartilhamento de instrumentos de avaliação e relevância da gratuidade das ferramentas para que professores e alunos possam usufruir das vantagens no âmbito educativo.

Por outro lado, entre as principais limitações encontradas, destacamos: credenciamento junto a instituições que gerenciam as ferramentas, idioma das ferramentas, bem como seus manuais, limitado à língua inglesa, necessidade de impressão de materiais, obrigação de investimento em equipamentos de hardware, uso exclusivo em determinados navegadores, falta de conhecimentos prévios para utilização e interface sobrecarregada de informações.

Tendo em conta as limitações encontradas, evidenciamos a relevância e necessidade de estudos futuros que possam analisar, de forma mais detalhada, as ferramentas apresentadas, assim como possibilidades de aplicação em sala de aula, com foco na avaliação dos recursos disponibilizados, por parte de docentes e estudantes, assim como o planejamento e criação de novas ferramentas que possam contribuir com a avaliação do processo de ensino-aprendizagem e na valoração de diferentes habilidades e competências, em diferentes modalidades formativas.

Antes de concluir, destacamos ainda a importância de o professor decidir entre as ferramentas elencadas, a que se adequa às suas necessidades de ensino, pois segundo Machado, Longhi e 
Behar (2013, p. 70), "o professor pode julgar o que é mais conveniente, interessante e motivador para o seu público-alvo, a fim de possibilitar a construção do conhecimento de forma dinâmica e significativa".

\section{Referências}

ANDRÉ, Ana Rita Dias; RODRIGUES, Ana Cláudia. Competência: conceito, evolução e desenvolvimento. In: COELHO, Iandra Maria Weirich da Silva. Competências no ensino-aprendizagem de línguas: pressupostos, práticas e reflexões. Campinas: Pontes Editores, 2019, p. 43-66.

ARBÓS, Albert. Evaluación de Competencias. Revista Panamericana de Pedagogía, v. 6, p. 27-47, 2005. Disponível em: https://bit.ly/2WxwYKR. Acesso em: 06 dez. 2020.

BARRA SALAZAR, A.; MORA DONOSO, M. Análisis de las metodologías de evaluación de los programas de formación basados en competencias. Revista Electrónica Actualidades Investigativas en Educación, v. 13, n. 3, 2013, p. 1-17. Disponível em: https://bit.ly/334BxyZ. Acesso em: 10 nov. 2020.

BEHAR, P. A. et al. Competências: conceito, elementos e recursos de suporte, mobilização e evolução. In: BEHAR, P. A. (Org.). Competências em Educação a Distância. Porto Alegre: Penso, 2013. p. 20-41.

BERROCOSO, J. V.; DOMÍNGUEZ, F. I. R.; SÁNCHEZ, M. R. F. Modelos de evaluación por competencias a través de un sistema de gestión de aprendizaje. Experiencias en la formación inicial del profesorado. Revista Iberoamericana de educación, v. 60, n. 1, p. 51-62, 2012. Disponível em: https://bit.ly/2Wzyv3o. Acesso em: 22 out. 2020.

BRANDÃO, F. S. R. Ferramenta Computacional de Apoio ao processo de avaliação das competências na Educação professional. 2012. 95 f. Dissertação (Mestrado Profissional em Computação Aplicada) - Instituto Federal de Educação, Ciência e Tecnologia do Ceará, Fortaleza, 2012. Disponível em: https://bit.ly/34hgVUd. Acesso em: 03 nov. 2020.

BRASIL. Base Nacional Comum Curricular. Brasília: MEC, 2017. Disponível em: https://bit.ly/2PzFWG5. Acesso em: 04 nov. 2020.

BRITO, Lélis Maia de et al. Ambientes virtuais de aprendizagem como ferramentas de apoio em cursos presenciais e a distância. RENOTE: revista novas tecnologias na educação. Porto Alegre, RS, 2013. Disponível em: https://bit.ly/354hy6z. Acesso em: 17 dez. 2020.

COELHO, I. M. W. da S. Introdução. In: COELHO, I. M. W. da S. (org) Competências no ensino-aprendizagem de línguas: pressupostos, práticas e reflexões. Campinas, SP: Pontes Editores, 2019. p.17-39.

COSTA, Luciano Andreatta Carvalho da; FRANCO, Sérgio Roberto Kieling. Ambientes virtuais de aprendizagem e suas possibilidades construtivistas. RENOTE: revista novas tecnologias na educação. Porto Alegre, RS, 2005. Disponível em: https://bit.ly/3rR0eM7. Acesso em: 17 dez. 2020.

COUTINHO, L. de A., Ferramenta para estimar a proficiência de indivíduos baseada na teoria de resposta ao item. 2013. 124 f. Dissertação (Mestrado em Engenharia da Computação e Sistemas). Universidade Estadual do Maranhão, São Luiz, 2013. Disponível em: https://bit.ly/2JCXOr2. Acesso em: 14 nov. 2020.

CRESWELL, J. W. Projeto de Pesquisa: Qualitativo, Quantitativo e mistos. 3 ed, Porto Alegre: Artmed, 2010.

DECLARAÇÃO DE BOLONHA, 1999. Disponível em: https://bit.ly/36lrQ15. Acesso em: 19 nov. 2020.

DIAS JÚNIOR, Maurício Vieira; MERCADO, Luis Paulo Leopoldo. Um instrumento para avaliação da aprendizagem em educação online a partir da análise de conteúdo. In: Simposio Internacional de Informática Educativa, 18., 2016, Porto. Anais... Porto. Disponível em: https://bit.ly/2LohYOn. Acesso em: 16 dez. 2020.

DÍAZ, F.; HERNÁNDEZ, G. Estrategias docentes para un aprendizaje significativo. México: McGraw-Hill, 2007. 
FEITOSA, M. P., Um modelo computacional de apoio à tomada de decisão em processos de avaliação de pessoas por competências. 2009, 83 f. Dissertação (Mestrado em Engenharia Elétrica e Computação) MACKENZIE, São Paulo, 2009. Disponível em: https://bit.ly/2X9wVpU. Acesso em 15 nov. 2019.

FERNÁNDEZ, A. M. La evaluación orientada al aprendizaje en un modelo de formación por competencias en la educación universitaria. REDU: Revista de Docencia Universitaria, v.1, n.8, 2010. Disponível em: https://bit.ly/33gl1M8. Acesso em: 31 dez. 2020.

FUENTES PENNA, Alejandro; CRUZ SÁNCHEZ, Ileana; DÍAZ DELGADO, David. Análisis comparativo de las plataformas tecnológicas para el estudio de posgrados en línea en México. Revista EDaPECI, São Cristóvão (SE), v.19, n.2, p. 40-51, 2019. Disponível em: https://bit.ly/2Zd1cEg. Acesso em: 10 nov. 2020.

GABARDO, Patricia; QUEVEDO, Silvia; ULBRICHT, Vânia Ribas. Estudo comparativo das plataformas de ensino-aprendizagem. Revista eletrônica de biblioteconomia e ciência da informação. Edição Especial, p.6584, 2010. Disponível em: https://bit.ly/2X9v58p. Acesso em: 10 nov. 2020.

GERRING, John. 2007. Case Study Research: Principles and Practice. Cambridge: Cambridge University Press.

GIL, A. C. Métodos e técnicas de pesquisa social. São Paulo Atlas, 2008.

GNU. O que é o software livre?. Disponível em: https://bit.ly/34niMqv. Acesso em: 01 nov. 2020.

Google Suite. Disponível em: https://bit.ly/2MxeBoG. Acesso em: 20 nov. 2020.

GONZÁLEZ-RUIZ, Sergio Luis et al. Una plataforma virtual para la evaluación e investigación on-line: Menpas. Cuadernos de Psicología del Deporte, v. 18, n. 3, p. 26-48, 2018. Disponível em: https://bit.ly/3b87rBm. Acesso em: 16 dez. 2020.

GRACIA, M. E. C.; ORTIZ, S. D. Herramientas TIC para la evaluación del aprendizaje, las competencias y las habilidades, de los alumnos del bachillerato. 2014. Disponível em: https://bit.ly/2Nyt00u. Acesso em: 01 out. 2020.

KLEMANN, M.; REATEGUI, E.; RAPKIEWICZ, C. Análise de Ferramentas de Mineração de Textos para Apoio à Produção Textual. In: Brazilian Symposium on Computers in Education, 2012. Disponível em: https://bit.ly/3oezAum. Acesso em: 13 nov. 2020.

MACHADO, Paula Maria Azevedo. O uso de plataformas educativas no ensino - aprendizagem de línguas: a plataforma MOODLE. 120 f. Dissertação de mestrado. Mestrado em Ensino do Português e Espanhol no $3^{\circ}$ Ciclo do Ensino Básico e Ensino Secundário, Universidade do Minho, Instituto de Educação, 2012. Disponível em: https://bit.ly/38anyN1. Acesso em: 12 nov. 2020.

MACHADO, L. R.; LONGHI, M. T.; BEHAR, P. A. Domínio Tecnológico: saberes e fazeres na educação a distância. In: BEHAR, Patricia Alejandra (Org). Competências em educação a distância. Penso Editora, 2013, p. 56-80.

MARINHO-ARAUJO, Claisy Maria; RABELO, Mauro Luiz. Avaliação educacional: a abordagem por competências. Avaliação, Campinas; Sorocaba, SP, v. 20, n. 2, 2015, p. 443-466. Disponível em: https://bit.ly/2X6phg2. Acesso em: 12 nov. 2020.

MARQUES, A.B.; BARBOSA, S.D.J.; CONTE, T. Representado a interação e navegação de sistemas interativos através de um modelo orientado à usabilidade: Um estudo de viabilidade. In: XV Simpósio Brasileiro sobre Fatores Humanos em Sistemas Computacionais (IHC 2016), São Paulo, 2016.

MEDEIROS, Matheus Ferreira; MEDEIROS, Alexsandro Melo. Educação e tecnologia: explorando o universo das plataformas digitais e startups na área da educação. V Conedu, Congresso Nacional de Educação, Anais..., Olinda, Pernambuco, 2018, p. 1-12. Disponível em: https://bit.ly/3hIWtn3. Acesso em: 10 dez. 2020. 
MORAIS, Nídia; CABRITA, Isabel. Ambiente Virtual de Aprendizagem num Contexto de b-learning. In: Conferência Internacional de Tecnologias de Informação e Comunicação na Educação, 5., 2007, Braga. Actas... Braga: p. 481-491. Disponível em: https://bit.ly/3rQeJ2B. Acesso em: 16 dez. 2020.

NIELSEN, J. LORANGER, H. Usabilidade na Web. Rio de Janeiro: Elsevier, 2007.

PASSERO, G.; ENGSTER, N. E. W.; DAZZI, R. L. S. Uma revisão sobre o uso das TICs na educação da Geração Z. RENOTE: revista novas tecnologias na educação, v. 14, n. 2, 2016. Disponível em: https://bit.ly/2JCVKEl. Acesso em 09 nov. 2020.

PERRENOUD, Philippe et al. As Competências para Ensinar no Século XXI: a formação dos Professores e o desafio da Avaliação. Tradução de Cláudia Schilling e Fátima Murad. Porto Alegre: Artmed Editora, 2002.

PÉREZ CERVANTES, M. L.; BARROS SAKER, A. F. Efectividad del uso de la plataforma virtual en el proceso de enseñanza y aprendizaje en la Universidad del Magdalena. Revista de Tecnología, v. 12, n. 3, p. 68-78, 2013. Disponível em: https://bit.ly/2MxeXM2. Acesso em: 16 dez. 2020.

PÉREZ-LIÑÁN, Aníbal. El método comparativo y el análisis de configuraciones causales. Revista Latinoamericana de Política Comparada, v. 3, 2010, p. 125-148. Disponível em: https://bit.ly/3nkmnPy. Acesso em: 16 dez. 2020.

PRIMO, L. P. C. A. Metodologia para acompanhamento de cursos de EAD e avaliação de competências A2COMP. 2004. 145 f. Dissertação (Mestrado em Informática Aplicada) - Universidade de Fortaleza, Fortaleza, 2004. Disponível em: https://bit.ly/2C0jL3Z. Acesso em: 10 nov. 2020.

SABOTA, Barbra; PEREIRA, Ariovaldo Lopes. O Uso de Ferramentas Tecnológicas em Ambientes de Aprendizagem: Critérios para Avaliação de Materiais de Ensino em Formato Digital. Caminhos em Linguística Aplicada, v. 16, n. 2, p. 44-62, 2017. Disponível em: https://bit.ly/3rS32Zo. Acesso em: 19 dez. 2020.

ROQUE, G. O. B.; ELIA, M.; MOTTA, C. L. R. da. Uma ferramenta para avaliação de competência baseada em desenvolvimento de projeto. In: SIMPÓSIO BRASILEIRO DE INFORMÁTICA NA EDUCAÇÃO, 9., 2004, Manaus. Anais... Manaus: UFAM, 2004. p.100-109. Disponível em: https://bit.ly/2X7PaMe. Acesso em: 10 mar. 2020.

SÁNCHEZ RODRÍGUEZ, José. Plataformas de enseñanza virtual para entornos educativos. Pixel-Bit. Revista de Medios y Educación, n. 34, 2009, p. 217-233. Disponível em: https://bit.ly/2JI5gJw. Acesso em: 16 dez. 2020.

SANTOS, J. R. A Moodle nas práticas pedagógicas de uma escola básica: realidade ou ficção na inserção das TIC em sala de aula. Educação, Formação \& Tecnologias, v. 5, n. 1, p. 72-83, 2012. Disponível em: https://bit.ly/2JE5r5i. Acesso em: 04 nov. 2020.

SILVA, Angela Carrancho da; LEITE, Ligia Silva; SILVA, Christina Marília Teixeira da. Avaliação da Aprendizagem em Ambientes Virtuais: é possível inovar?. Meta: Avaliação, v. 1, n. 2, p. 237-248, 2009. Disponível em: https://bit.ly/2MpA8zq. Acesso em: 16 dez. 2020.

TOVAR-GÁLVEZ, J. C. et al. Concepción, formación y evaluación por competencias: reflexiones en torno a posibles alternativas pedagógicas y didácticas. Educação \& Sociedade, v. 33, n. 121, p. 1257-1273, 2012. Disponível em: https://bit.ly/335hRLp. Acesso em: 15 nov. 2020.

Recebido em janeiro de 2020.

Aprovado para publicação em dezembro de 2020.

\section{Gilmara Oliveira Maquiné}

Mestra em Ensino Tecnológico - Instituto Federal de Educação, Ciência e Tecnologia do Amazonas - IFAM, Brasil

gilmaramaquine@gmail.com

\section{Iandra Maria Weirich da Silva Coelho}

Doutora e Mestre em Linguística - Docente do Instituto Federal de Educação, Ciência e Tecnologia do Amazonas - IFAM, Brasil

iandrawcoelho@gmail.com 